\title{
An investigation of channel flow with a smooth air-water interface
}

\author{
Reza Madad • John Elsnab - Cheng Chin · Joseph Klewicki • Ivan Marusic
}

Received: date / Accepted: date

\begin{abstract}
Experiments and numerical simulation are used to investigate fully developed laminar and turbulent channel flow with an air-water interface as the lower boundary condition. Laser Doppler velocimetry measurements of streamwise and wall-normal velocity components are made over a range of Reynolds number based upon channel height and bulk velocity from 1100 to 4300, which encompasses the laminar, transitional and low Reynolds numbers turbulent regimes. The results show that the airflow statistics near the stationary wall are not significantly altered by the air-water moving interface, and reflect those found in channel flows. The mean statistics on the water interface side largely exhibit results similar to simulated Poiseuille-Couette flow (PCF) with a solid moving wall. For second order statistics, however, the simulation and experimental results show some discrepancies near the moving water surface, suggesting that a full two-phase simulation is required. A momentum and energy transport tubes analysis is investigated for laminar and turbulent PCFs. This analysis builds upon the classical notion of a streamtube and indicates that part of the energy from the pressure gradient is transported towards the stationary wall, and is dissipated as heat inside the energy tubes, while the remainder is transmitted to the moving wall. For the experiments, the airflow energy is transmitted towards the water to overcome the drag force and drive the water forward; therefore, the amount of energy transferred to the
\end{abstract}

Reza Madad · John Elsnab · Cheng Chin · Joseph Klewicki · Ivan Marusic

Department of Mechanical Engineering, University of Melbourne,

Parkville, Victoria 3010, Australia

E-mail: john.elsnab@unimelb.edu.au

Joseph Klewicki

Department of Mechanical Engineering, University of New Hamp-

shire,

Durham, New Hampshire 03824, USA water is higher than the energy transferred to a solid moving wall.

\section{Introduction}

Numerous engineering applications involve an interaction between a gas flowing adjacent to a moving liquid. This interaction has been investigated extensively due to its application in the petroleum, chemical and nuclear industries as well as in geophysical and environmental sciences (Banerjee, 2007). Despite the efforts in this area of research, there remains an incomplete understanding of turbulent gas motion near a liquid interface. This arises from difficulties in experimentally measuring or numerically simulating velocity fields in the vicinity of moving surfaces, and is further complicated by the turbulent nature of the flow in most applications of practical interest. The investigation of this flow (concurrent gas-liquid flow) is considerably complicated by the two-phase boundary condition. In this study, we test how well a simplified Poiseuille-Coutte flow can be used to act as a proxy for the real two-phase flow, and in doing so, clarify aspects of the underlying physics.

A number of experimental studies have looked at the interaction between a turbulent airflow and a water film flowing parallel to it. Only a few, however, have looked at the present configuration, which is an air-water channel. Hanratty and Engen (1957) conducted experiments in such a channel to examine the interaction between a turbulent air stream above a moving water film. Their results indicated no distortion in the airflow velocity profile for a smooth water surface and for a water surface with ripples. Hanratty and Engen (1957) and Dykhno et al (1994) measured the air velocity profiles and found that the maximal velocity shifts $10-12 \%$ towards the stationary wall. These trends display a significantly different behaviour with those observed by 
later researchers. For example, both Paras et al (1998) and Wongwises and Kalinitchenko (2002) measured the air velocity profiles and found that the maximal velocity shifts toward the interface. The inconclusive results of this basic statistical quantity suggests that further study is warranted.

The objective of this research is to determine the effects of an air-water interaction on the airflow statistics near the interface and stationary wall in a (nominally) fully developed channel flow. The streamwise and wall-normal velocity components are obtained using two-component laser Doppler velocimetry (LDV). A surrogate direct numerical simulation (DNS) of a Poiseulle-Coutte flow is also performed using the experimentally obtained interface velocity as the boundary condition at $\delta^{+}=135$, where $\delta^{+}=\delta U_{\tau} / \nu$, $\delta$ is the half channel height, $U_{\tau}$ is the friction velocity, and $v$ is the kinematic viscosity. This allows one to comment on the accuracy of a reduced simulation, which is a substantially cheaper computation to perform in comparison to a two-phase simulation. These comparisons also provide insights into the underlying flow physics. The flow physics of the two-phase flow and PCF are further clarified using a momentum and energy transport tube analysis. Meyers and Meneveau (2013) describe the properties of momentum and energy transport tubes, which are analogous to a streamtube.

\section{Experimental Details}

\subsection{Experimental Facility}

The experiments were conducted in the facility schematically shown in Fig. 1. The air height, $H$, is $24.9 \mathrm{~mm}$ with an aspect ratio of 11 . The depth of the water beneath the air flow was $350 \mathrm{~mm}$. The aspect ratio is sufficient to ensure a nominally two-dimensional flow (Dean, 1978). Air is drawn through the diffuser using a suction fan. Water circulation was nominally promoted by a fixed speed pump in the direction of airflow. The pump alone produces a surface velocity of approximately $0.02 \mathrm{~m} / \mathrm{s}$. The concurrent flow in combination with a weir generates a stable moving air-water interface that was primarily driven by the airflow itself. Water enters the tank from a $32 \mathrm{~mm}$ diameter PVC pipe that is split using two $80 \mathrm{~mm}$ diameter pipes. The water then passes through two mesh screens and a honeycomb section to ensure water flow uniformity at the channel inlet. The surface velocity of the water flow was quantified using a video camera to track insoluble Polyethylene particles floating on the surface (specific gravity of $0.95-0.96$ ). Also, it was observed that the pump (without running the fan) produces a water surface oscillation of less than $\pm 0.15 \mathrm{~mm}(0.5 \% \mathrm{H})$ and equates to $0.5-1.5$ viscous units.

\subsection{LDV Measurements}

The LDV system was calibrated using two methods: a spinning disk (Park et al, 2002) and a spinning wire (Bean and Hall, 1999; Kurihara et al, 2002). The preferred method of calibration for a two-component LDV system is a spinning disk. The measurement volume is focused onto the surface of a roughened disk that is driven by a servo-motor. The horizontal $(U)$ and vertical $(V)$ components of velocity were calibrated by positioning the measurement volume at $90^{\circ}$ and $0^{\circ}$, respectively. The total uncertainty in both components was approximately $0.5 \%$. A spinning wire was used to ensure that the LDV system sampling rate of each channel is correct. A significant difference between the spinning wire and disk methods is that only a single particle (e.g., wire tip) passes through the probe volume per revolution in comparison to multiple particles. The nominal standard deviation in the sampling rate over a range in angular velocity from 300 to 600 RPM for the $U$ and $V$ components is 0.0016 and $0.00191 / s$, respectively.

The LDV measurement volume had dimensions of $0.085 \mathrm{~mm}$ in height, $0.085 \mathrm{~mm}$ in width and $1.2 \mathrm{~mm}$ in length for the blue beam $(488.0 \mathrm{~nm})$, and $0.089 \mathrm{~mm}$ in height, $0.089 \mathrm{~mm}$ in width, and $1.3 \mathrm{~mm}$ in length for the green beam $(514.5 \mathrm{~nm})$ (Kurihara et al, 2002). The measurement volume in terms of viscous units was $\Delta_{z}^{+} \simeq 7, \Delta_{y}^{+} \simeq 0.5$, and $\Delta_{x}^{+} \simeq 0.5$ at $\delta^{+}=131$. Further details regarding the LDV measurement volume, calibration methods, and results can be found in Madad (2013).

\subsection{Inner Normalization and Scaling}

Scaling analysis of turbulence quantities requires an estimate of the wall shear stress, $\tau_{w}$. The friction velocity is derived from the wall shear stress, $U_{\tau}=\sqrt{\tau_{w} / \rho}$, where $\rho$ is the fluid density. For fully developed, two-dimensional Poiseuille-Couette flow, use of the mean statement of momentum conservation yields,

$\tau_{m w}-\tau_{s w}=H \frac{d p}{d x}$

where $\tau_{m w}=\left.\mu \frac{d u}{d y}\right|_{m w}$ and $\tau_{s w}=\left.\mu \frac{d u}{d y}\right|_{s w}$ are the wall shear stress at the moving wall and stationary wall, respectively, $\mu$ is the dynamic viscosity, $p$ is the mean pressure, and $x$ is the streamwise axis. The contribution from the vertical sidewalls contributes to the overall pressure drop in Eq. 1, but is negligible since the aspect ratio is greater than 10 . The friction velocity for the stationary wall, $U_{\tau_{s w}}$, and moving wall, $U_{\tau_{m w}}$, are determined using a four point linear curvefit of $U$ within the linear sublayer. The uncertainty in the balance between the left-hand-side and right-hand-side of Eq. 1 is less than $7 \%$. 


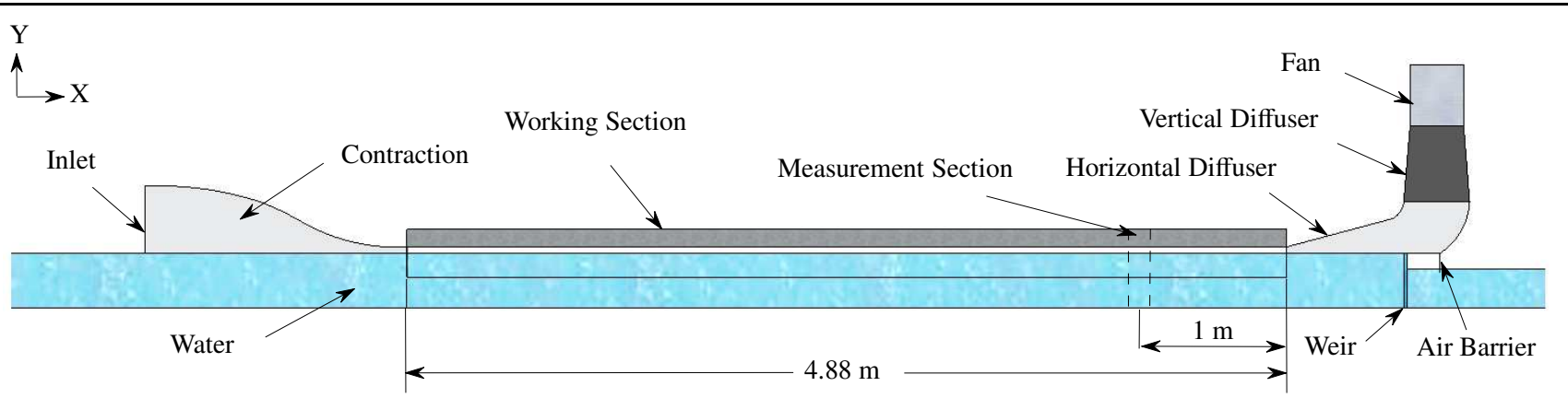

Fig. 1 Schematic of the experimental set-up.

El Telbany and Reynolds (1980) suggested the moving wall velocity scaling is likely to be proportional to $(y / H)^{-0.5}$ and $\gamma^{-1}$, where $\gamma$, is the ratio of the Couette $\left(H U_{m w} / v\right)$ to Poiseuille $\left(H U_{b} / v\right)$ flow Reynolds number, $U_{b}$ is the bulk velocity, and $y$ is the distance from the moving wall. In this study the following relationship proposed by Thurlow and Klewicki (2000) is applied to obtain the local friction velocity of the moving wall

$U_{\tau_{M W}}=\frac{U_{\tau_{m w}}}{1-5 \gamma \sqrt{y / H}}$

Using $U_{\tau_{M W}}$ improves the collapse of the data in comparison to $U_{\tau_{m w}}$. Note that for the present analysis, this only changes $U_{\tau_{m w}}$ on average by $4.6 \%$.

\subsection{Experimental Parameters}

The experimental data were measured at a fixed downstream distance of $170 \mathrm{H}$ from the inlet. This distance exceeds that required for fully developed flow $(130 H)$ (Lien et al, 2004; Monty, 2005) for the case of solid wall channels. In the present case the airflow can be considered streamwise homogeneous to a very good approximation. Since the LDV settings are the same for all the measurements, the spatial resolution degrades slightly with increasing $\delta^{+}$. For parameter range and symbol definition, see table 1 . The lowest Reynolds number, $R e=H U_{b} / v=1170\left(\delta^{+}=40.4\right)$, is in the laminar regime and the remainder are in the transitionally turbulent regime with a smooth moving water surface. The highest Reynolds number, $R e=4267\left(\delta^{+}=140\right)$, corresponds to the first appearance of ripples on the water surface.

\subsection{Poiseuille-Couette Flow Simulation}

The direct numerical simulation of Poiseuille-Couette flow is based upon a spectral element/ Fourier spatial discretization with a second-order velocity-correction projection scheme

\begin{tabular}{cccccc}
\hline \hline$R e$ & $\delta^{+}{ }_{s w}$ & $\delta^{+}{ }_{m w}$ & $U_{\tau_{s w}}(m / s)$ & $U_{\tau m w}(m / s)$ & Symbol \\
1170 & 40.4 & 40.4 & 0.051 & 0.050 & $\times$ \\
2237 & 76.0 & 75.1 & 0.087 & 0.086 & $\nabla$ \\
2581 & 86.9 & 84.0 & 0.101 & 0.098 & + \\
2958 & 101 & 98.7 & 0.102 & 0.100 & $\diamond$ \\
3683 & 118 & 113 & 0.129 & 0.123 & $\triangleleft$ \\
3710 & 125 & 118 & 0.142 & 0.134 & $\square$ \\
3836 & 131 & 124 & 0.144 & 0.139 & $\circ$ \\
4003 & 135 & 132 & 0.146 & 0.142 & $\Delta$ \\
4267 & 144 & 138 & 0.167 & 0.160 & $\star$ \\
\hline
\end{tabular}

Table 1 Parameters and symbol definition used in the data presentation. Note that the subscript $s w$ and $m w$ denote stationary and moving wall, respectively.

for the temporal discretization as described by Blackburn and Sherwin (2004). The Fourier coordinate requires geometric homogeneity in the spanwise direction, while the remaining planar geometry is discretized using nodal spectral elements with a Gauss-Lobatto-Legendre local mesh in each element. A 10th order Legendre polynomial is employed within each spectral element. The computational domain length in the streamwise is $L_{x}=30 \pi \delta$, the spanwise width is $L_{z}=3 \pi \delta$, and the domain height is $2 \delta$. This volumetric domain consists of $1280 \times 256 \times 200$ collocation points in the streamwise, spanwise and wall-normal directions, respectively. There are at least 40 grid points within the buffer region, $y^{+}=y U_{\tau} / v \approx 30$, with 10 grid points within $y^{+} \leq 0.675$. The chosen domain length is sufficient for the convergence of the statistics considered in this work according to Chin et al (2010). For this simulation, the timestep is $\Delta t=0.001$ (dimensionless) and the collection of statistics is initiated after sufficient time (a minimum of 5 wash-through times based on the bulk flow speed and domain length) has elapsed to allow the flow to reach a statistically steady state. The turbulence statistics are then averaged over 20 wash-through times to achieve convergence. The simulation parameters are: $\Delta x^{+}=9.94, \Delta y_{\text {wall }}^{+}=0.075$, $\Delta y_{\text {centre }}^{+}=3.15$, and $\Delta z^{+}=4.97$. The fully developed turbulent PCF simulation is conducted at a Reynolds number of $\delta^{+}=135$. The fixed velocity of the moving water surface 
obtained from the experimental results is used as the boundary condition. At $\delta^{+}=135$, the velocity at the air-water interface is $U_{m w}=0.12 \mathrm{~m} / \mathrm{s}$.

\section{Results}

\subsection{Mean Velocity Profiles}

The mean velocity profiles normalized by the maximum velocity, $U_{m}$, over the Reynolds number range of $40<\delta^{+}<$ 145 are presented in Fig. 2a. The analytical solution for laminar Poiseuille-Couette flow is in agreement with the measured data at $\delta^{+}=40$. As expected, the experimental data show that the mean velocity profiles flatten in the non-laminar regime. A comparison of the experimental mean velocity profile at $\delta^{+}=135$ and simulated PCF is shown in Fig. 2 b. The simulation data match the experimental data near the stationary wall $(0<y / H<0.12)$ and reasonably well over the remainder of the channel height.

\subsection{Skin Friction}

The coefficient of friction, $C_{f}$, for pure Poiseulle flows is obtained using Dean's equation (Dean, 1978) and is given as

$C_{f}=0.073 R e^{-0.25}$

The $C_{f}$ values obtained from Eq. 3 and the experimental data for the stationary wall/moving water surface are shown in Fig. 3. Very good agreement is seen between the stationary wall friction coefficients and the results of Dean's empirical equation. As expected from PCF with a forward moving wall e.g., (Thurlow and Klewicki, 2000), the values of $C_{f}$ for the moving side are lower than the stationary side; here, $C_{f_{m w}}$ is on average about $5 \%$ lower $C_{f_{s w}}$.

\subsection{Inner Normalized Mean Velocity Profiles}

The inner-normalized mean velocity profiles for the stationary side and the moving side (using $U_{\tau_{s w}}$ and $U_{\tau_{M W}}$, respectively) are shown in Figs. $4 a$ and 4 b, respectively. For the moving side, the water surface velocity is subtracted from the mean velocities. These profiles merge onto a single curve for $y^{+} \leq 20$ and collapse well on the $U^{+}=y^{+}$curve within the viscous sublayer (Fig. 4). Beyond $y^{+}=30$, the higher Reynolds number profiles approach a logarithmic-like dependence. These trends are similar to the results of Elsnab et al (2011) for post-laminar channel flows over a range of low Reynolds numbers.
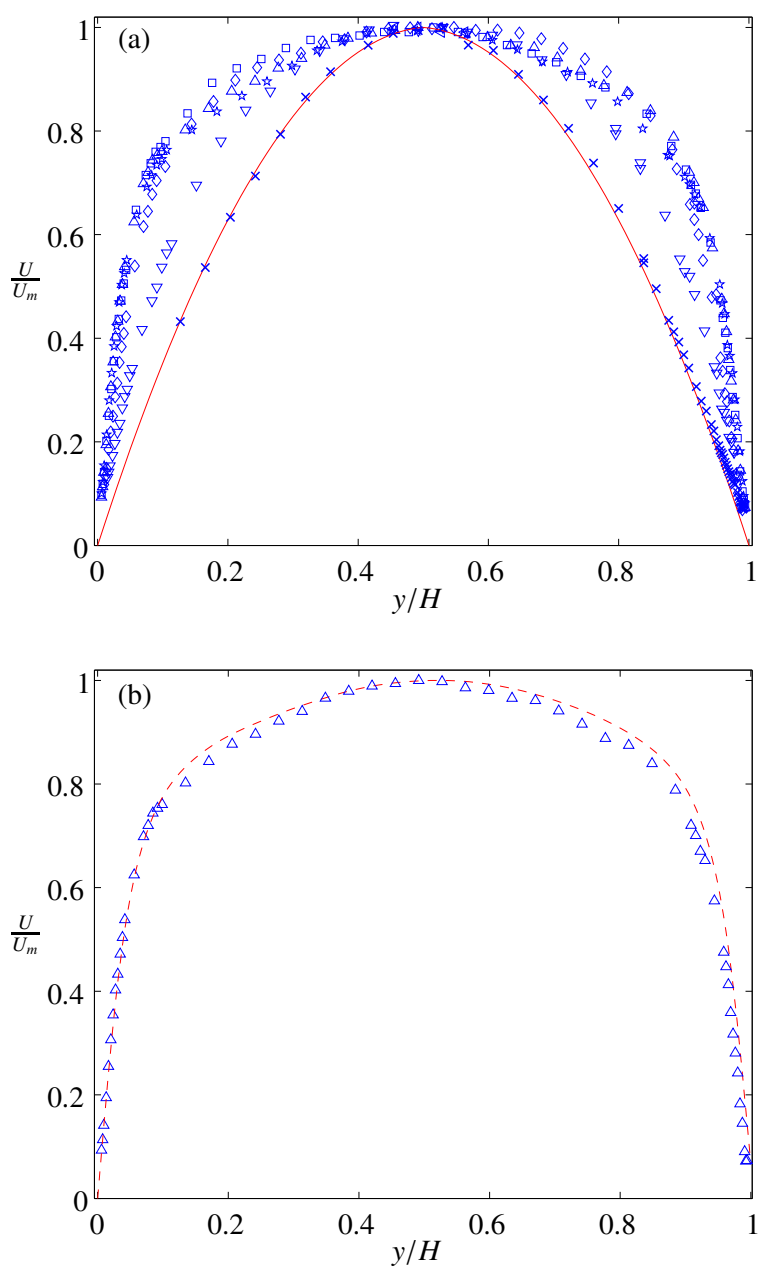

Fig. 2 Normalized mean velocity profiles for $40<\delta^{+}<145$ (a). The symbol definition is presented in table 1 and the solid line corresponds to laminar PCF theory where the stationary wall is at $y / H=0$. A comparison between the mean velocity profile from the experiment and simulated PCF (dashed line) at $\delta^{+}=135$ is shown in (b).

Comparisons between the inner-normalized velocity profiles from the experiment and simulated PCF for the stationary and moving side are shown in Fig. $5 \mathrm{a}$ and $5 \mathrm{~b}$, respectively, as well as DNS channel flow at matched $\delta^{+}$. The mean velocity profile obtained from the experiments and DNS nominally adhere to inner-scaling in the near-wall region and core flow. Also, the water motion does not have an appreciable effect on the stationary wall velocity profile. This observation is consistent with previous PCF studies (e.g., El Telbany and Reynolds (1980) and Thurlow and Klewicki (2000)). For a fixed $\delta^{+}$, both the stationary wall and moving wall sides inner normalized velocity profiles are in good agreement with DNS channel flow data by Laadhari (2002) provided that the wall velocity is subtracted from the moving wall data. 


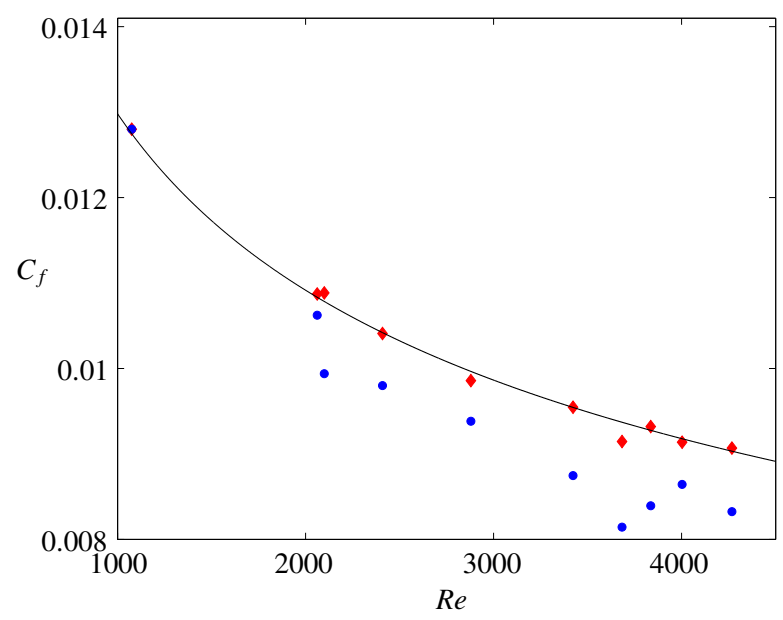

Fig. 3 Skin friction coefficient values for $1100<R e<4300$ from the experiment. Description: $\downarrow$, stationary wall $C_{f}$; $\bullet$, moving water wall $C_{f}$; and solid line, Eq. 3 (Dean, 1978).

\subsection{Turbulent Velocity Fluctuations}

The root-mean-square (rms) streamwise velocity fluctuations, $u_{r m s}$, for the stationary side is presented in Fig. 6a. The peak position in $u_{r m s}$ for all experimental results is located at $y^{+} \approx$ 15 , and the maximum value is on average about $5 \%$ lower than DNS profiles of Tsukahara et al (2005) at $\delta^{+}=80$ and 110. The $u_{r m s}$ for the moving side are shown in Fig. 6b. The position of the peak $u_{r m s}$ for the water side is $y^{+} \approx 17$ and the maximum value is about $4 \%$ lower than for the present stationary wall data (see Fig. 8a). These results are in good agreement with the observations of Thurlow and Klewicki (2000) and Spencer et al (2009).

The rms wall-normal velocity fluctuations, $v_{r m s}$ for the stationary wall along with channel flow DNS results by Tsukahara et al (2005) at $\delta^{+}=80$ and 110 are shown in Fig. 7a, and for the moving side in Fig. 7b. The variation in $v_{r m s}$ from the stationary wall $(y / H=0)$ towards the moving water surface is shown in Fig. 8b. The peak wall-normal turbulence intensity near the air-water interface $(y / H=1)$ at higher Reynolds numbers, $124 \leq \delta^{+} \leq 144$, is higher than near the stationary wall $(y / H=0)$. However, this is not the case for lower Reynolds numbers, $76 \leq \delta^{+} \leq 118$. For $76 \leq \delta^{+} \leq 118$, the maximum value of $v_{r m s}$ remains the same near the stationary and moving surfaces and show similar behaviour to Poiseuille flow. For $124 \leq \delta^{+} \leq 144$, the maximum value near the stationary wall is lower than the interface value. The effect of the moving water on $v_{r m s}^{+}$ does not agree with PCF studies and becomes more significant with increasing Reynolds number. A possible explanation for this observation is the physical difference between a solid moving wall and an air-water interface (presence of
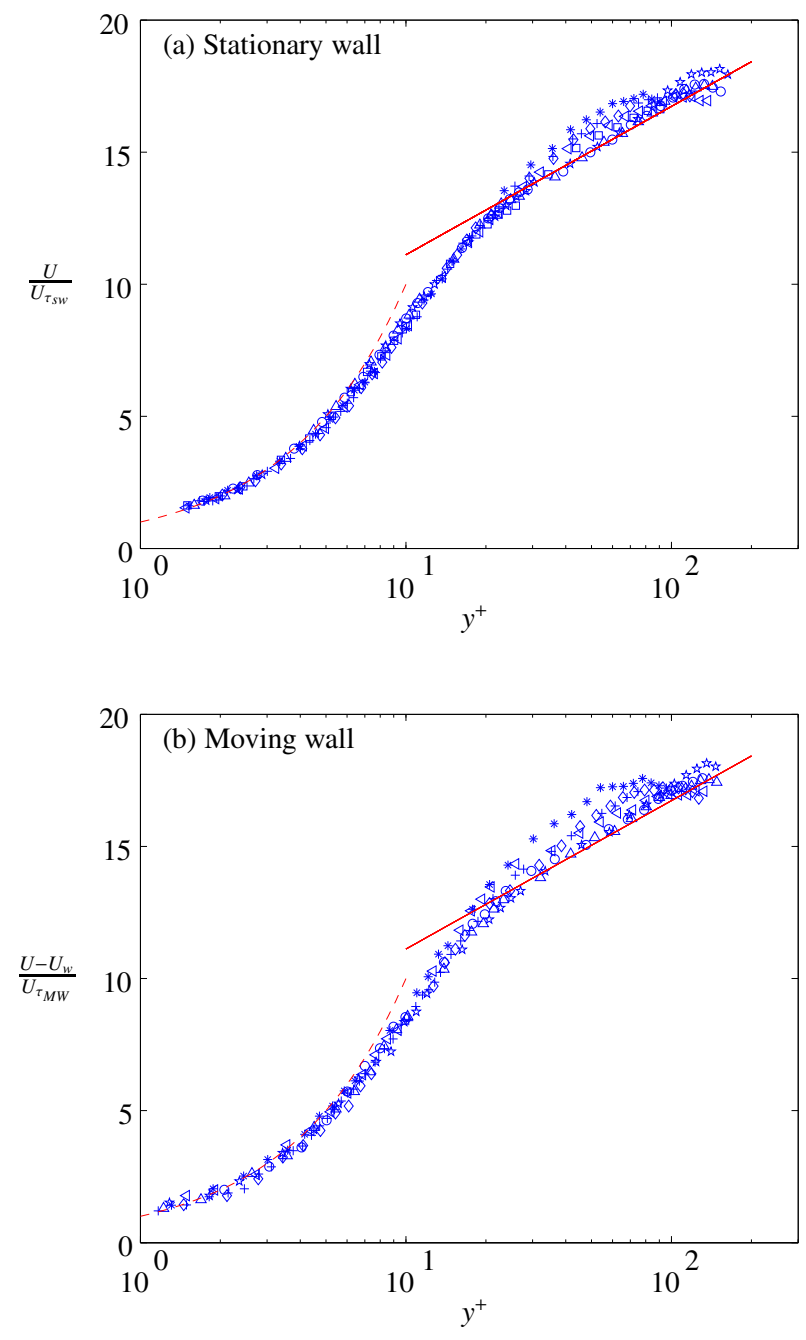

Fig. 4 Inner-normalized mean velocity profiles for the stationary wall (a) and for the moving water surface (b). Description: symbols, see table 1, dashed-line, $U^{+}=y^{+}$, and the solid line, $U^{+}=1 / 0.41 \ln \left(y^{+}\right)+$ 5.5 .

water surface oscillation). Because the air-water interface is compliant and not shear-free, the parallel surface fluctuations are not zero due to the slip boundary condition, as they are for a solid surface. Additionally, because the interface is compliant and only kept flat by surface tension, the normal fluctuations can be larger near the interface. This, of course, depends on the relative strength of the interface stresses as compared to the turbulent pressure fluctuations.

The streamwise rms velocity fluctuation profiles of the simulated PCF and current experiment at $\delta^{+}=135$ are essentially identical for the stationary side, with the peak near the stationary wall located at $y^{+}=14$ and 16 for the simulation and experiment, respectively. Close to the moving water surface, however, the peak appears farther from the moving wall for the experiment $\left(y^{+}=20\right)$ than for the simu- 

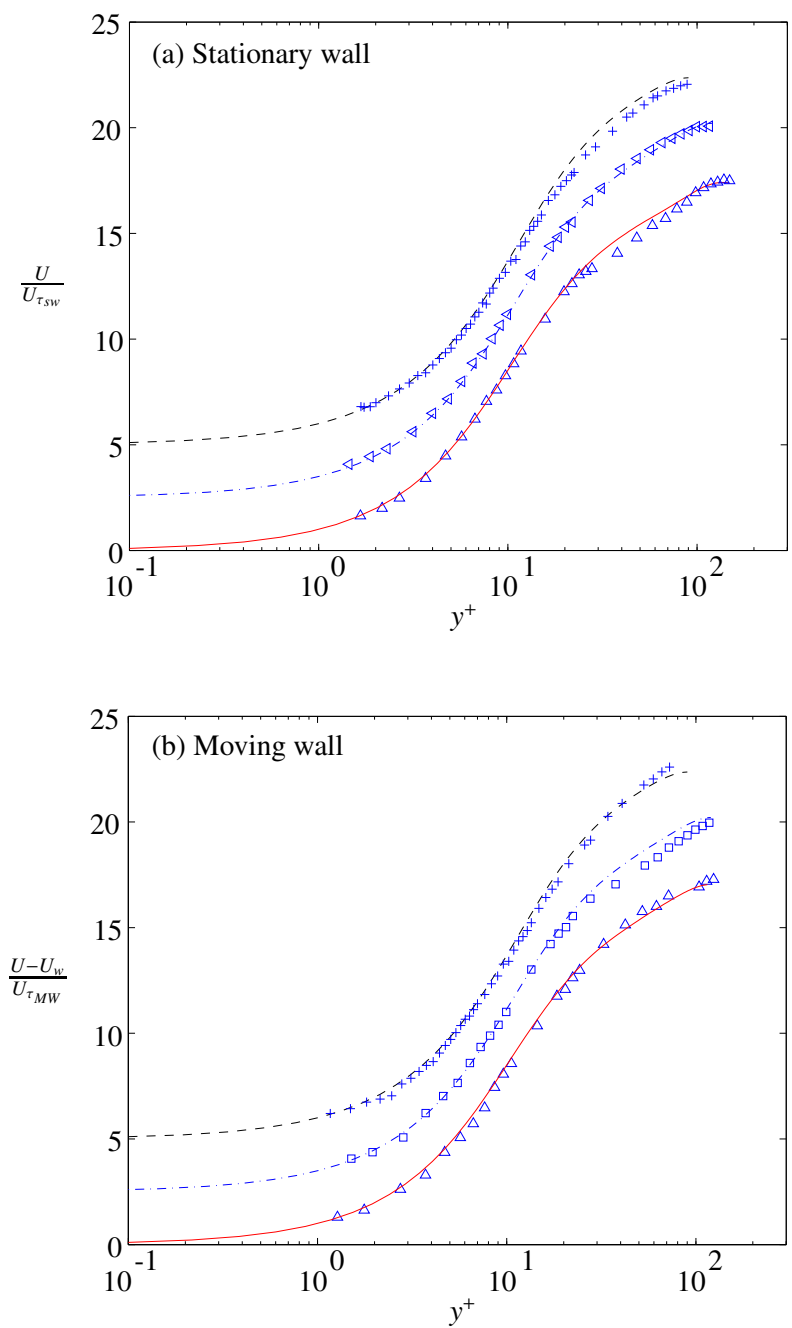

Fig. 5 Velocity profile of the stationary side (a) and the moving side (b). Description: symbols, see table 1, solid line, simulation at $\delta^{+}=$ 135 and channel flow DNS by Laadhari (2002) where the dash-dot line is at $\delta^{+}=90$ and the dashed line is at $\delta^{+}=120$. Note that the profiles are shifted 2.5 viscous units for clarity.

lation $\left(y^{+}=15\right)$. The peak of $v_{r m s}^{+}$near the stationary wall is higher than the peak near the moving wall for the simulation, while the opposite occurs for the experiment at $\delta^{+}=135$. The overall features of the experimentally derived rms profiles and their differences with the simulation are clarified in Figs. $8 \mathrm{a}$ and $\mathrm{b}$.

\subsection{Reynolds Stress}

The inner-normalized Reynolds stress profiles for both stationary wall and moving water side are shown in Fig. 9. The Reynolds stress increases in peak value and the shifts outward in viscous units with increasing Reynolds number for both the stationary and moving water side of the channel.
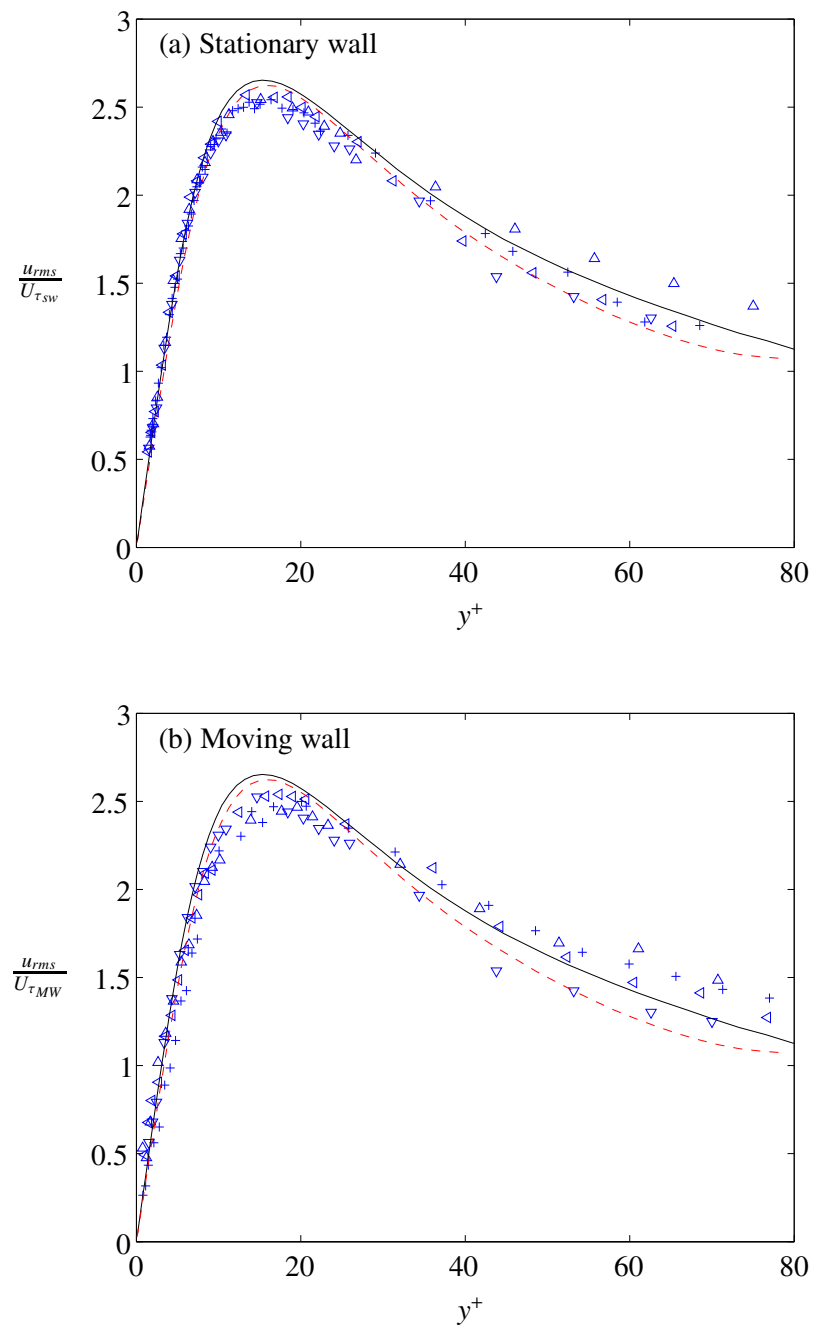

Fig. 6 RMS velocity fluctuations of the streamwise velocity. Description: dashed-line, $\delta^{+}=80$, solid line, $\delta^{+}=110$ are from DNS channel flow data by Tsukahara et al (2005); and symbol definition, see table 1 .

The Reynolds stress varies linearly from the peak towards the centreline for the stationary side and is nearly linear for the moving water side. This linear variation is consistent with channel flow results, which can be derived from classical analysis of the once-integrated mean momentum equation. For the moving side, the experimental data at $\delta^{+}=135$ shows much higher Reynolds stress from the core region to the buffer layer (corresponding to $0.5<y / H<0.9$ ) compared to simulated PCF. Water level oscillations may at least partly underlie this rather dramatic difference.

\subsection{Turbulence Kinetic Energy Production}

The turbulent kinetic energy production, $E_{p}{ }^{+}(y)=-\langle u v\rangle^{+}$ $\partial U^{+} / \partial y^{+}$, is a dominant term in the kinetic energy budget. 

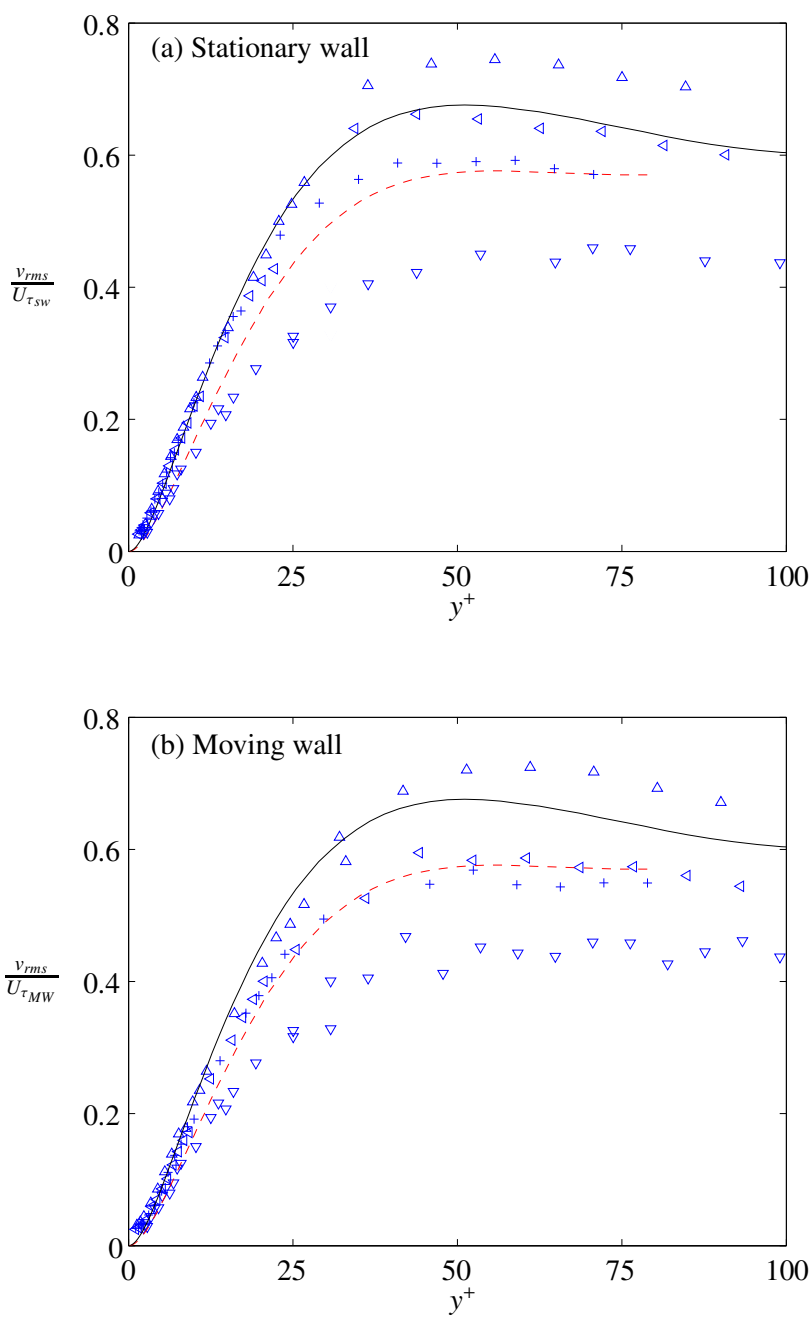

Fig. 7 Root-mean-square velocity fluctuations of wall-normal velocity. Description: dashed-line, $\delta^{+}=80$, solid line, $\delta^{+}=110$ are from DNS channel flow data by Tsukahara et al (2005); and symbol definition, see table 1

Profiles of $E_{p}^{+}$normalized by the stationary wall friction velocity for the experiment and simulation are shown in Fig. 10. Both profiles exhibit consistent results with diminishing energy production close to the channel centreline and peaks near both walls with amplification of $E_{p}^{+}$near the stationary wall and attenuation near the moving wall. Spencer et al (2009) also observed a similar trend, as the energy production is lower close to the co-moving wall.

It is also of interest to consider the difference between the energy production profiles of experimental and simulated results for half of the channel towards the moving wall $(0.5<y / H<1)$. Although the peak near the moving wall (DNS) is higher than the peak near the moving water surface (experiment), the area under both profiles is approximately the same, and the net energy production of the DNS is about
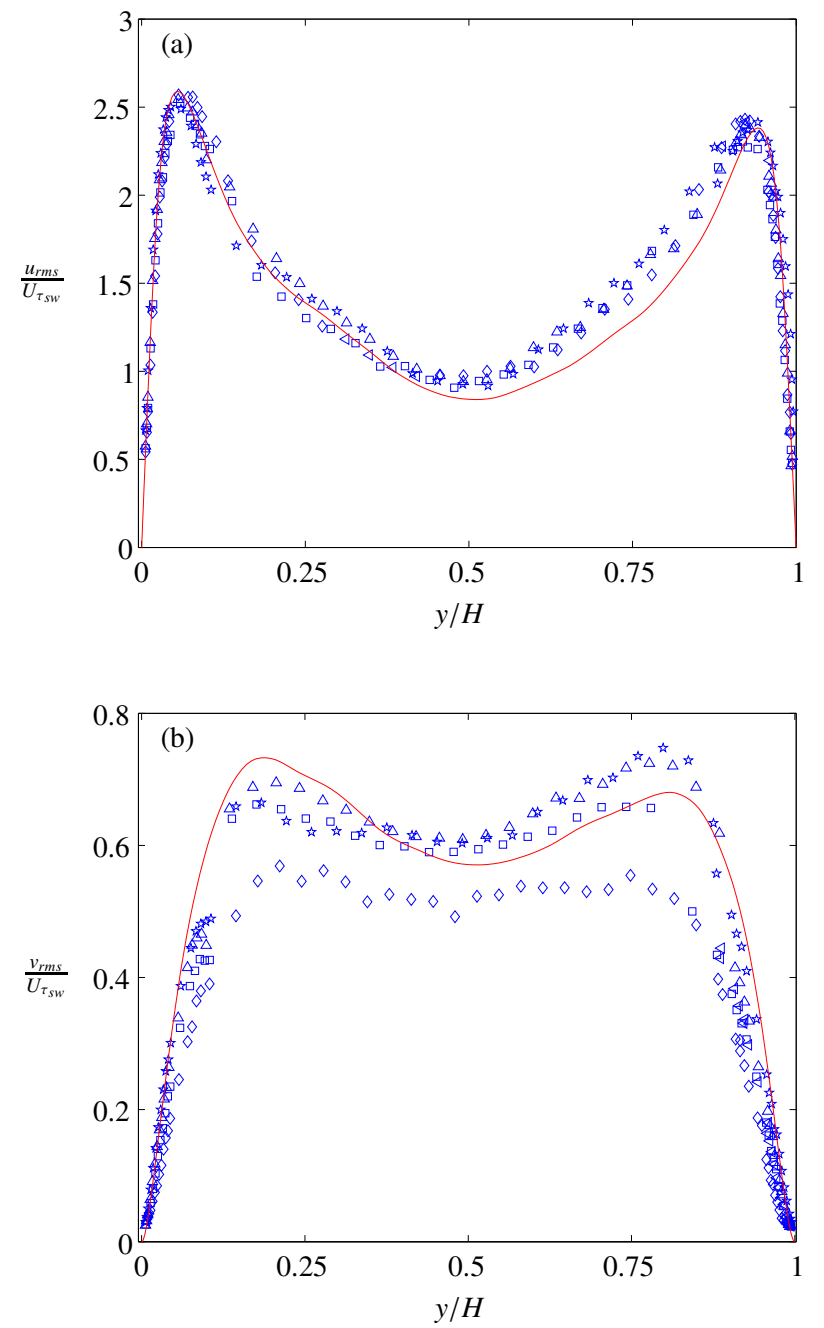

Fig. 8 Root-mean-square velocity fluctuations of streamwise velocity (a) and root-mean-square velocity fluctuations of wall-normal velocity (b) where $y / H=0$ is the stationary wall. Description: solid line corresponds to the simulation at $\delta^{+}=135$ and the symbol definition is presented in table 1 .

$5 \%$ larger than the experiment, which is attributed to the difference in $\langle u v\rangle^{+}$velocities since the mean velocity gradient is essentially symmetric across the channel.

\subsection{Transport of Momentum and Energy}

Momentum and energy transport can be graphically depicted using transport tube analyses, which builds upon the classical notion of a streamtube. Meyers and Meneveau (2013) used these concepts to visualize turbulent flow processes associated with wind turbine arrays. There is no exchange of momentum or energy through the corresponding tube mantle. Momentum and energy transport tubes, like streamtubes, are not Galilean invariant. The similarity between classi- 

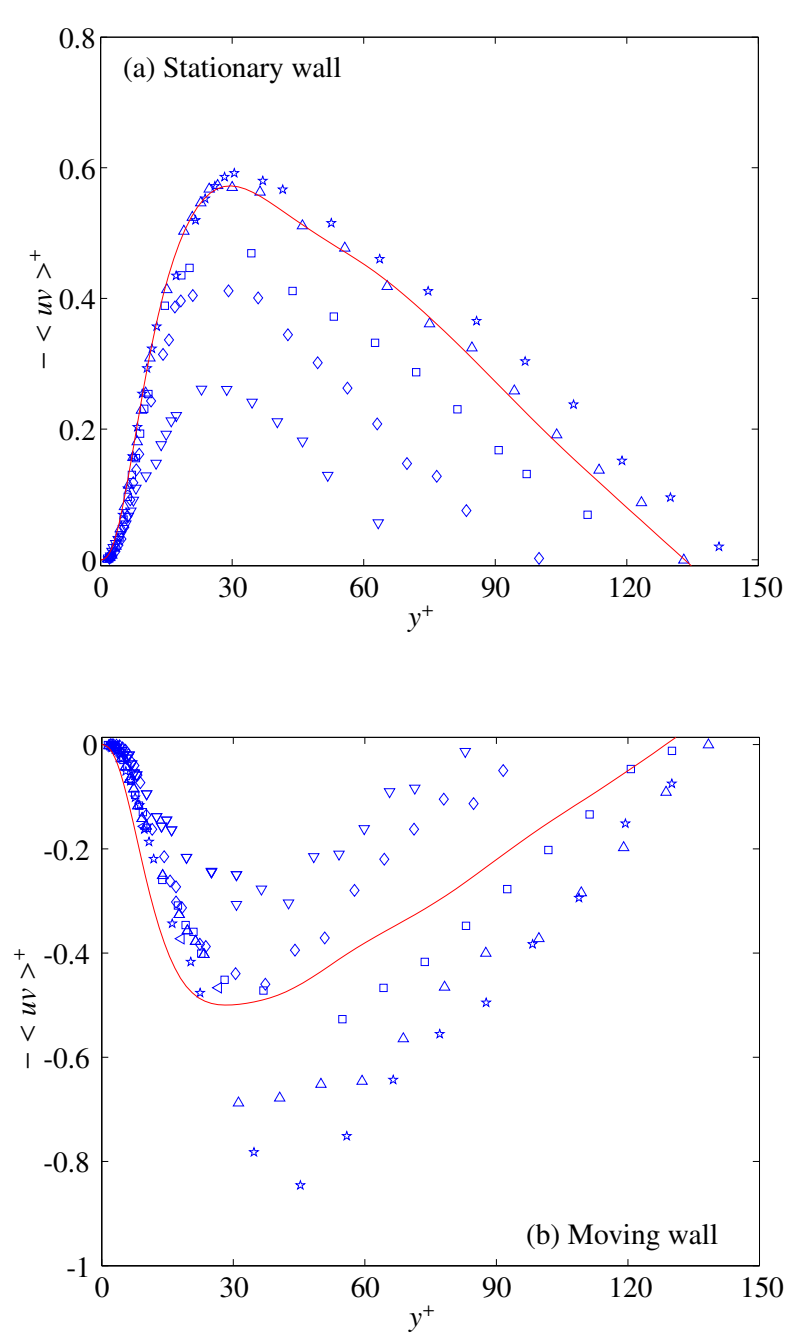

Fig. 9 Inner-normalized Reynolds stress profiles for the stationary wall (a) and for the moving water surface (b). Symbol definition is presented in table 1 and the solid line corresponds to the simulation at $\delta^{+}=135$.

cal streamtubes and the momentum/energy transport tubes is that the flux is constant across sections except for integral effects of sinks and sources due to viscous dissipation and turbulent kinetic energy production. For steady, inviscid flow, streamtubes, momentum and energy transport tubes all coincide since the viscous and Reynolds stress are zero. However, for turbulent flows, momentum tubes can be significantly different from the energy transport tubes due to the Reynolds stresses. The combination of laminar Couette and Poiseuille flows is of interest because its structure provides a basis for better understanding of momentum and energy transport in the presence of a no-slip and a moving wall. The velocity profile for laminar PCF is $U=(d p / d x)\left(y^{2}-\right.$ $y H) /(2 \mu)+y U_{m w} / H$. Using the velocity profile, the two components of momentum flux are

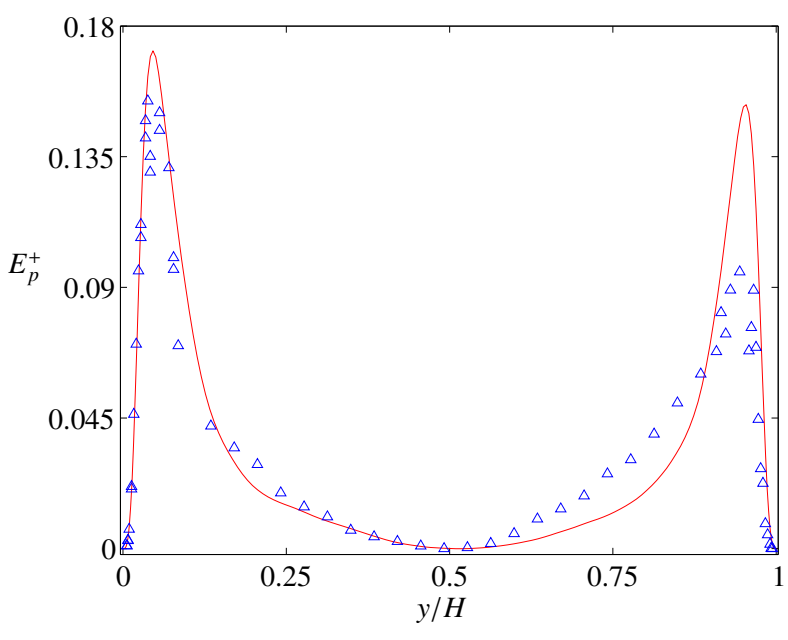

Fig. 10 Inner normalized turbulent kinetic energy production profiles from the experiment and simulation. The solid line corresponds to the simulation at $\delta^{+}=135$ and $y / H=0$ is the stationary wall.

$$
\begin{aligned}
& \bar{F}_{1}=U^{2}=\left(\frac{d p}{d x} \frac{y(y-1)}{2 \mu}+y U_{m w}\right)^{2} \\
& \bar{F}_{2}=-v \frac{\partial U}{\partial y}=-v\left(\frac{1}{2 \mu} \frac{d p}{d x}(2 y-1)+U_{m w}\right)
\end{aligned}
$$

where it is assumed $H=1$. Integration of $d y / d x=\overline{F_{2}} / \overline{F_{1}}$ and rearrangement allows the momentum lines, $x=y(x)$, to be written as

$$
\begin{aligned}
& \qquad x=-\frac{C}{8 v} y^{4}+\frac{C-U_{m w}}{4 v} y^{3}-\frac{\left(C-U_{m w}\right)^{2}}{16 C v} y^{2} \\
& -\frac{\left(C-U_{m w}\right)^{3}}{16 C^{2} v} y-\frac{\left(C-U_{m w}\right)^{4}}{32 C^{3} v} \ln \left(U_{m w}-C+2 C y\right) \\
& \text { where } C=(d p / d x) /(2 \mu) .
\end{aligned}
$$

The momentum transfer lines for different moving wall velocities are constructed using Eq. 6. The velocity profiles and corresponding momentum lines for different PCFs normalized with the moving wall velocity for a constant pressure gradient are shown in Fig. 11. The evolution of the momentum transport lines from "Poiseuille-type flow" (a) to "Couette-type flow" (d) are shown in these figures. The momentum added in the bulk flow through the pressure gradient is transported towards the sidewalls because the pressure gradient dominates. However, the location of the zero momentum transport line, $Y_{Z C M=0}$, continually moves towards the moving wall with increasing wall velocity. At the zero momentum transport line, the total advection of momentum occurs horizontally. By increasing the moving wall velocity, the effect of Couette flow dominates over the pressure 

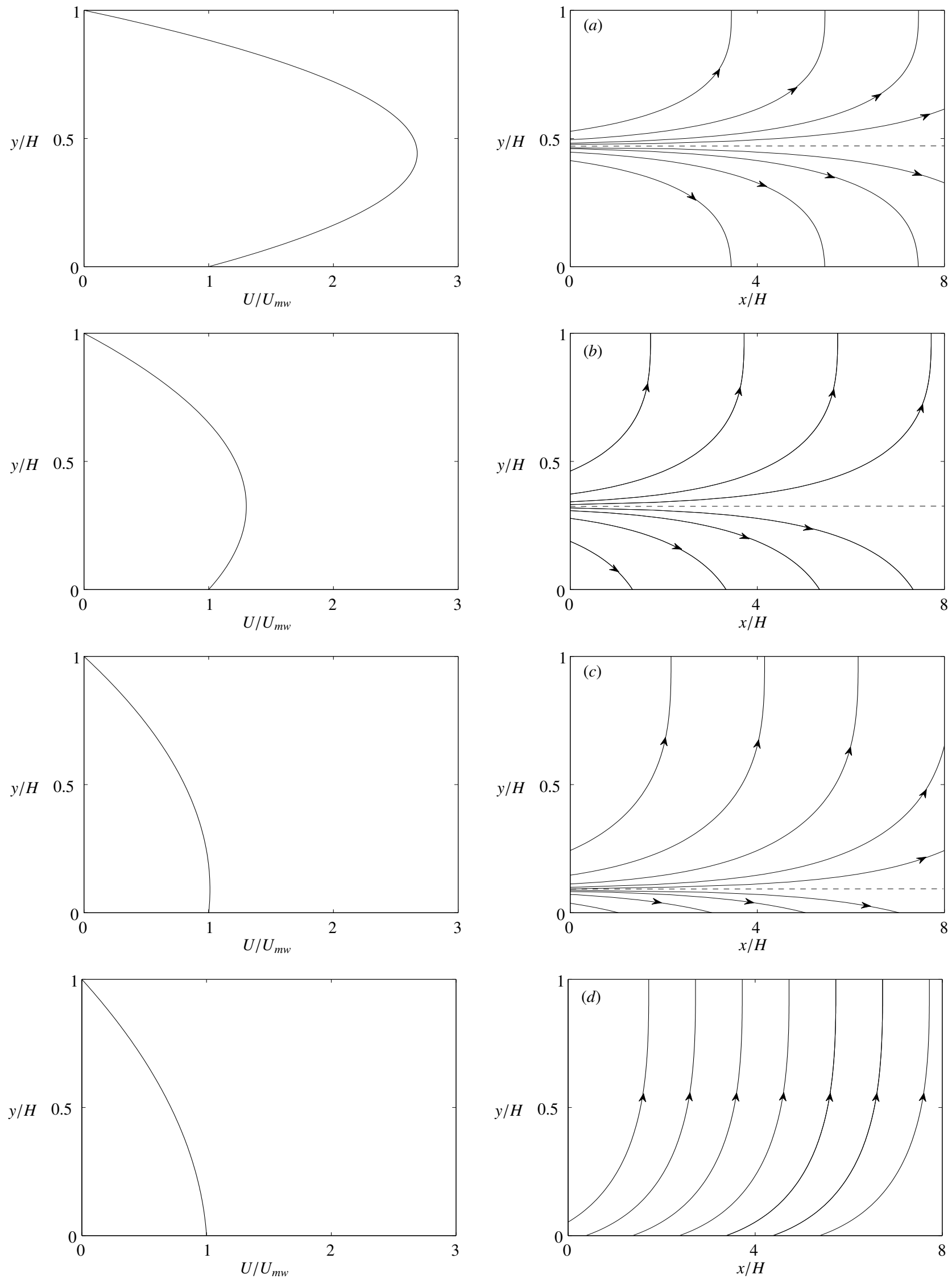

Fig. 11 Velocity profiles and momentum lines of laminar PCF with different moving wall velocity ((a) through (d) corresponds to the different velocity profiles shown at the left). The dashed-line is the zero momentum transport line. $y=1$ is the stationary wall. Note that it is assumed $H=1$. 
gradient and the momentum lines eventually form a profile similar to pure Couette flow.

The energy transport lines for laminar flow have similar shape as the momentum lines, but twice the slope. The interpretation for energy tubes for Poiseuille- and Couette-type flows are as follows: for Poiseuille-type flow (Fig. 11a), the kinetic energy of the pressure gradient is dissipated as heat inside the tubes towards the stationary wall (top wall) and transferred to the moving wall (bottom wall). By increasing the moving wall speed, a larger part of the energy from the pressure gradient is transferred to the moving wall and converted to the work done by the moving wall. The rest of the energy is dissipated as heat inside the mantle and is directed towards the stationary wall (see Figs. 11b, c). For the Couette-type flow (Fig. 11d) the work done by the moving wall dominates over the pressure gradient, and the energy of moving wall is transferred entirely towards the stationary wall. This energy is dissipated as heat inside the energy tubes before reaching the stationary wall.

The momentum lines for the laminar airflow PCF solution and the laminar experimental data are shown in Fig. 12. The momentum lines for the analytical and experimental results are similar, although the location of the zero momentum transport line from the experimental data moves slightly towards the moving water $(y \approx 0.47 H)$, in comparison to the analytical solution $(y \approx 0.5 H)$.

As indicated with arrows in Fig. 12, the momentum added to the bulk flow through the pressure gradient is transferred towards the stationary top wall and the moving water surface (bottom wall). The effect of the pressure gradient dominates over the effect of the moving wall (Couette effect), and the momentum lines of the current experiment are similar to the laminar pure Poiseuille flow. However, the location of the zero momentum transport line for the experimental results moves slightly towards the moving water surface. The momentum added by the pressure gradient to the airflow is transmitted to the water, which in turn drives the water forward. The momentum from the moving water surface is then transported towards the stationary bottom wall of the water tank over a large streamwise distance $(110 H)$.

\subsubsection{Turbulent Flow}

Using the results presented in Meyers and Meneveau (2013), the two components of the energy flux for the turbulent airflow are formed as follows:

$$
\begin{aligned}
& \bar{F}_{1}=\frac{U^{3}}{2}+U<u u> \\
& \bar{F}_{2}=U\left(<u v>-v \frac{\partial U}{\partial y}\right)
\end{aligned}
$$
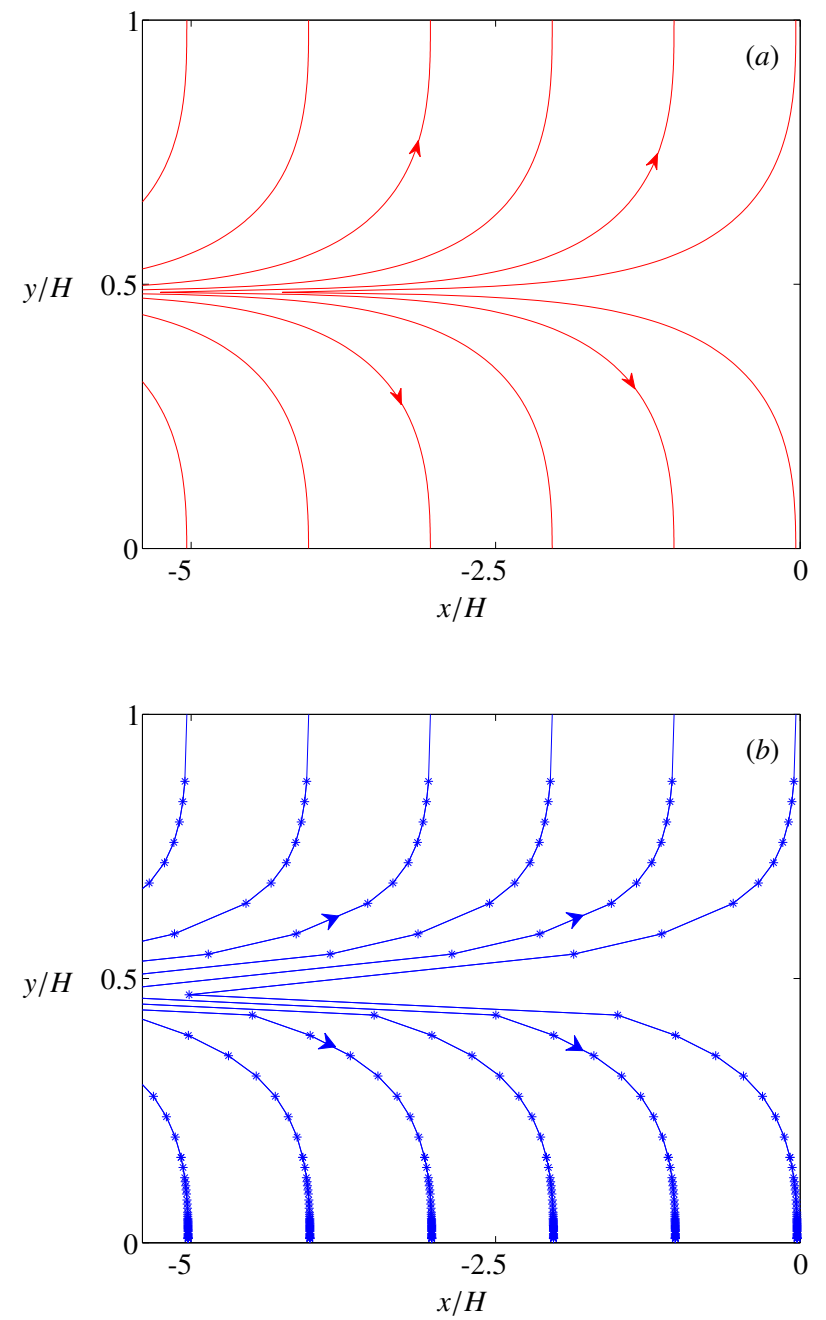

Fig. 12 The momentum lines of the laminar airflow. Analytical solution for laminar PCF (a) and experimental data of the airflow with a moving water surface (b), where $y / H=1$ is the stationary wall.

Hence, the slopes of the tangent lines of this vector field are given by:

$\frac{d y}{d x}=\frac{\bar{F}_{2}}{\bar{F}_{1}}=\frac{<u v>-v \frac{\partial U}{\partial y}}{\frac{U^{2}}{2}+<u u>}$

Numerical integration yields an energy line for turbulent airflow with one stationary wall and a moving water surface. The energy lines of the experiment and simulation are shown in Fig. 13. The data in this figure reveals that the energy line of the simulated Poiseuille-Couette flow with a moving solid wall for this Reynolds number is similar to pure Poiseuille flow since the ratio of Couette to Poiseuille Reynolds number is low $(\gamma=0.07)$. In addition, the location of the zero energy transport line (no vertical transport) is at the channel's centerline. However, the energy line of the current ex- 
periment shows its location moves significantly towards the moving wall $(y / H \approx 0.3)$. Near both the stationary and the moving wall, the transport lines become vertical as more of the energy transport occurs through viscous diffusion. Close to the stationary wall, this vertical energy line is about $0.05 H$ from the wall. Both the DNS and experimental data show the same trend. The simulated PCF data show that near the moving wall the vertical line extends the same distance into the flow. In contrast, the experimental data show that this line extends much farther $(0.2 H)$ from the moving water surface.
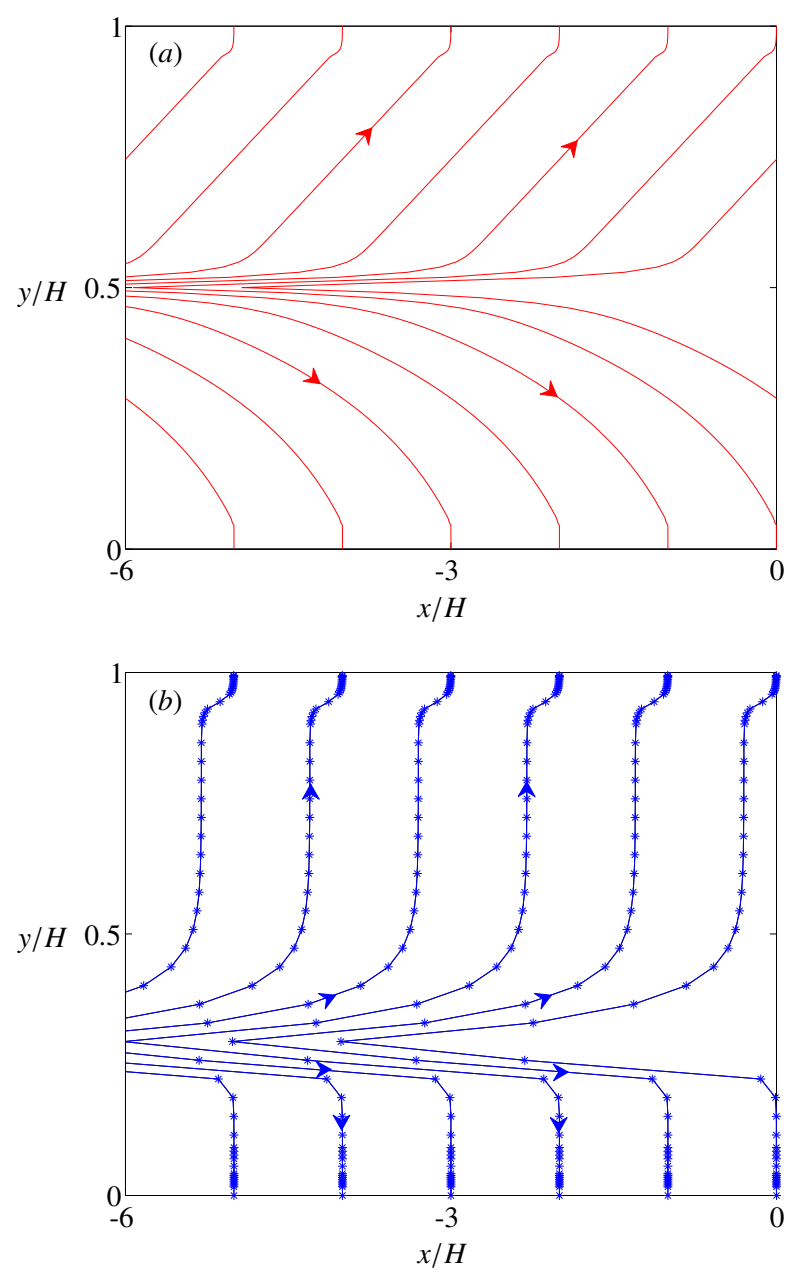

Fig. 13 Energy lines of airflow for simulated data (a) and experimental data (b) where $y / H=1$ is the stationary wall.

\section{Concluding Remarks}

The results show that over the range of Reynolds numbers investigated, the airflow statistics near the stationary wall are not effected by the air-water moving interface and are comparable with pure Poiseuille flows. Also, a number of the statistics on the moving side show results similar to the simulated PCF with a solid moving wall. It can be concluded that, in order to simulate airflow within a fixed wall and a moving water with a smooth surface (no waves), there is minimal need to simulate water flow underneath the interface to obtain nominally accurate mean airflow statistics at a fixed streamwise distance, i.e., a moving water surface can be simulated as a solid moving wall. However, for second order statistics, a two-phase simulation (from bottom wall of the water tank to top wall of the air channel) is required, as simulation and experimental results have discrepancies near the moving water surface. Here, momentum and energy tube analysis reveals that these discrepancies are due to a greater consumption of the airflow energy to overcome the water drag and drive the water forward, as compared to the PCF configuration with a moving solid wall.

Despite these differences in momentum and energy at the surface, an interesting question remains as to why the mean statistics for the two-phase flow still agree reasonably well with the PCF case. Here we speculate that surface tension may spatially localize the effect of the boundary condition. Essentially, the water surface behaves like an elastic sheet due to force from surface tension. The ratio of the viscous to surface tension forces is the capillary number $(\mathrm{Ca})$. At $\delta^{+}=135$, it is estimated that $\mathrm{Ca}<0.1$ (characteristic length is the fully developed length of the flow $2.44 \mathrm{~m}$ ), therefore, surface tension forces are greater than viscous forces acting on the water surface. When surface tension dominants, a good approximation for the mean flow is obtained by modelling the two-phase flow as PCF. As $\delta^{+}$ increases, ripples form on the water surface indicating the gravity effects become important, i.e., $U / U_{b}=f(\operatorname{Re}, \mathrm{Ca}, \mathrm{Fr})$, where $F r$ is the Froude number. Clearly this becomes an increasingly complicated flow that requires further investigation.

Acknowledgements The authors gratefully acknowledge the support of the Australian Research Council. We would also like to thank Dr Laadhari and Dr Tsukahara for making their DNS data available. We are also grateful to make this contribution in celebration of Lex Smits' illustrious career.

\section{References}

Banerjee S (2007) Transport at the Air-Sea Interface. Springer Berlin Heidelberg

Bean VE, Hall JM (1999) New primary standards for air speed measurement at NIST. Proceedings of NCSL Workshop pp Virginia, USA

Blackburn HM, Sherwin SJ (2004) Formulation of a Galerkin spectral element-Fourier method for three- 
dimensional incompressible flows in cylindrical geometries. J Comput Phys 197:759

Chin C, Ooi A, Marusic I, Blackburn H (2010) The influence of pipe length on turbulence statistics computed from direct numerical simulation data. Phys Fluids 22:115,107

Dean RB (1978) Reynolds number dependence of skin friction and other bulk flow variables in two-dimensional rectangular duct flow. J Fluids Eng 100:215-223

Dykhno LA, Williams LR, Hanratty TJ (1994) Maps of mean gas velocity for stratified flows with and without atomization. Int J Multiphase Flow 20:691-702

El Telbany MMM, Reynolds AJ (1980) Velocity distribution in plane turbulent channel flows. J Fluids Mech 100:1

Elsnab J, Klewicki J, Maynes D, Ameel T (2011) Mean dynamics of transitional channel flow. J Fluid Mech 678:451-481

Hanratty TJ, Engen JM (1957) Interaction between a turbulent air stream and a moving water surface. AIChE J 3:299-304

Kurihara N, Terao Y, Takamoto M (2002) LDV calibration for the air speed standard between 1.3 to $40 \mathrm{~m} / \mathrm{s} .5^{\text {th }}$ International Symposium on Fluid Flow Measurement 61:Virginia, USA

Laadhari F (2002) On the evolution of maximum turbulent kinetic energy production in a channel flow. Phys Fluids 14:L65-L68

Lien K, Monty JP, Chong MS, Ooi A (2004) The entrance length for fully developed turbulent channel flow. Proceedings of the 15th Australasian Fluid Mechanics Conference

Madad R (2013) An experimental investigation of channel flow with a smooth air-water interface. $\mathrm{PhD}$ thesis, The University of Melbourne, Australia

Meyers J, Meneveau C (2013) Flow visualisation using momentum and energy transport tubes and applications to turbulent flow in wind farms. J Fluid Mech 715:335-358

Monty JP (2005) Developments in smooth wall turbulent duct flows. PhD thesis, The University of Melbourne, Australia

Paras SV, Vlachos NA, Karabelas AJ (1998) LDA measurements of local velocities inside the gas phase in horizontal stratified atomization two-phase flow. Int J Multiphase Flow 24:651-661

Park JT, Cutbirth JM, Brewer WH (2002) Hydrodynamic performance of the Large Cavitation Channel (LCC). Technical Report NSWCCD-50-TR 68:California, USA

Spencer NB, Lee LL, Parthasarathy RN, Papavassiliou DV (2009) Turbulence structure for plane Poiseuille Couette flow and implications for drag reduction over surfaces with slip. Can J Chem 87:38-46

Thurlow EM, Klewicki J (2000) Experimental study of turbulent Poiseuille Couette flow. Phys Fluids 12:865
Tsukahara T, Seki Y, Kawamura H, Tochio D (2005) DNS of turbulent channel flow at very low Reynolds numbers. In Proc of the Forth Int Symp on Turbulence and Shear Flow Phenomena Williamsburg, USA:935-940

Wongwises S, Kalinitchenko V (2002) Mean velocity distributions in a horizontal air-water flow. Int J Multiphase Flow 28:167-174 


\section{University Library}

\section{- M M N E R VA A gateway to Melbourne's research publications}

Minerva Access is the Institutional Repository of The University of Melbourne

Author/s:

Madad, R;Elsnab, J;Chin, C;Klewicki, J;Marusic, I

Title:

An investigation of channel flow with a smooth air-water interface

Date:

2015-06-01

Citation:

Madad, R., Elsnab, J., Chin, C., Klewicki, J. \& Marusic, I. (2015). An investigation of channel flow with a smooth air-water interface. Experiments in Fluids, 56 (6), https://doi.org/10.1007/ s00348-015-1985-8.

Persistent Link:

http://hdl.handle.net/11343/55194 